\title{
Multi-valued backward stochastic differential equations with regime switching
}

\section{Ruijuan Deng ${ }^{1 *}$ (D) and Yong Ren ${ }^{2}$}

\section{"Correspondence:}

ruijuandeng@163.com

'Department of Basic, Wuhu

Institute of Technology, Wuhu,

China

Full list of author information is

available at the end of the article

\section{Springer}

\begin{abstract}
The paper considers a class of multi-valued backward stochastic differential equations with subdifferential of a lower semi-continuous convex function with regime switching, whose generator is a continuous-time Markov chain with a finite state space. Firstly, we get the existence and uniqueness of the solution by the penalization method. Secondly, we prove that the solution of the original system is weakly convergent. Finally, we give an application to the homogenization of a class of multi-valued PDEs with Markov chain.
\end{abstract}

Keywords: Multi-valued; Existence and uniqueness; Penalization method; Weakly convergent

\section{Introduction}

In recent years, many researchers have done a lot of interesting work on nonlinear backward stochastic differential equations (BSDEs, in short) with different generators.

As an important mathematical tool in probability theory, Markov chain has vast applications in diverse fields. One can see Siu [1] for more details. From the numerical point of view, diffusions are generally approximated by Markov chains. Thus, there is a great motivation to discuss Markov chain systems. Based on these facts, Lu and Ren [2] considered a class of mean-field BSDEs based on finite-state, continuous time Makov chain. Tao et al. [3] proposed a class of BSDEs coupled with a finite state Markov chain, which has two-time scale structure:

$$
Y_{t}=\xi+\int_{t}^{T} f\left(s, \alpha_{s}, Y_{s}\right) d s-\int_{t}^{T} Z_{s} d B_{s}-\sum_{j \in I} \int_{t}^{T} W_{s}(j) d \tilde{\mathcal{V}}_{s}(j)
$$

Based on Meyer-Zheng topology, they showed that the solution is weakly convergent. In that paper, the corresponding reaction-diffusion equations were explained in the sense of viscosity solution, and the convergence of PDEs was proved.

In recent years, the development of BSDEs has been very rapid. The extension forms of BSDEs are various. Wu and Zhang [4] focused on BDSDEs which are locally monotone assumptions. At the end of that paper, the Sobolev weak solutions for a kind of SPDEs were given. This conclusion greatly broadens the applicability of this kind of equations. Some conclusions obtained in BSDEs are also widely used in other fields. Wei et al. [5] dealt

(c) The Author(s) 2019. This article is distributed under the terms of the Creative Commons Attribution 4.0 International License (http://creativecommons.org/licenses/by/4.0/), which permits unrestricted use, distribution, and reproduction in any medium, provided you give appropriate credit to the original author(s) and the source, provide a link to the Creative Commons license, and indicate if changes were made. 
with the Sobolev weak solution of HJB equation, in which the nonlinear Doob-Meyer decomposition theorem obtained from the BSDEs is the main contributor.

In particular, Pardoux and Răşcanu [6] gave some results about multi-valued BSDEs (MBSDEs, in short). In that paper, they presented a probabilistic interpretation for the viscosity solution of some parabolic and elliptic variational inequalities. Recently, there have been many interesting developments about MBSDEs, one can see Yang et al. [7], Guo [8], Malinowski [9], etc. These achievements have enriched the theoretical system of MBSDEs, and some of them also gave relevant applications.

Under the framework, we continue to discuss the class of multi-valued BSDEs as follows:

$$
Y_{t}=\xi+\int_{t}^{T} f\left(s, \alpha_{s}, Y_{s}, Z_{s}, W_{s}\right) d s-\int_{t}^{T} Z_{s} d B_{s}-\sum_{j \in I} \int_{t}^{T} W_{s}(j) d \tilde{\mathcal{V}}_{s}(j)-\int_{t}^{T} U_{s} d s,
$$

in which the function $f$ not only relates to the process $Y$, but also relates to the processes $Z$ and $W$. What is more, the function $f$ here contains a Markov chain.

Firstly, we do some preparation for follow-up certification. Secondly, we give the main results of this paper. Then, we prove the weak convergence result under the Meyer-Zheng topology. Finally, we give the homogenization of a class of multi-valued PDEs with Markov chain.

\section{Basic assumptions, preliminaries, and notations}

At the beginning of the paper, we introduce some foundations of the follow-up discussion, such as the definition of multi-valued BSDEs, notations, assumptions, and so on.

Suppose that $(\Omega, \mathcal{F}, \mathbb{P})$ is a probability space, $\left\{B_{t}, t \in[0, T]\right\}$ is a $d$-dimensional Brownian motion, $\left\{\alpha_{t}, t \in[0, T]\right\}$ is a finite state Markov chain, and the state space is $I=\{1,2, \ldots, m\}$, in which $m$ is a positive integer. The transition intensities are $\lambda_{i j}(t)$ for $i \neq j$, which is nonnegative and bounded. And $\lambda_{i i}(t)=-\sum_{j \in I \backslash i i\}} \lambda_{i j}(t)$. Suppose that $\mathbb{F}=\left(\mathcal{F}_{t}\right)_{t} \in[0, T]$ is a filtration which is generated by $\left\{B_{s}, \alpha_{s} ; s \in[0, T]\right\}$ and augmented by all $\mathbb{P}$-null sets of $\mathcal{F}$.

In this paper, $\mathcal{V}_{t}(j)$ is the number of jumps of $\left\{\alpha_{s}\right\}$, and $\phi$ is a lower semi-continuous convex function defined on $\mathbb{R}$. More details can be found in [3].

The multi-valued BSDEs with the Markov chain are defined as follows.

Definition 1 The solution is a quadruple of $\left(Y_{t}, Z_{t}, U_{t}, W_{t}\right)_{0 \leq t \leq T}$ of progressively measurable processes, which takes values in $\mathbb{R} \times \mathbb{R}^{1 \times d} \times \mathbb{R} \times \mathbb{R}^{m} \rightarrow \mathbb{R}$ and satisfies that:

(i) $\mathbb{E}\left(\sup _{0 \leq t \leq T}\left|Y_{t}\right|^{2}\right)<\infty, \mathbb{E} \int_{0}^{T}\left|Z_{t}\right|^{2} d t<\infty, \mathbb{E} \int_{0}^{T}\left|W_{t}(j)\right|^{2} \mathbf{1}_{\left\{\alpha_{t-\neq j\}}\right.} \lambda_{\alpha_{t-j}}(t) d t<\infty$, $\mathbb{E} \int_{0}^{T}\left|U_{t}\right|^{2} d t<\infty$

(ii) For all $0 \leq t \leq T$, it holds that

$$
\begin{aligned}
Y_{t}= & \xi+\int_{t}^{T} f\left(s, \alpha_{s}, Y_{s}, Z_{s}, W_{s}\right) d s-\int_{t}^{T} Z_{s} d B_{s} \\
& -\sum_{j \in I} \int_{t}^{T} W_{s}(j) d \tilde{\mathcal{V}}_{s}(j)-\int_{t}^{T} U_{s} d s ;
\end{aligned}
$$

(iii) $\mathbb{E} \int_{0}^{T} \phi\left(Y_{s}\right) d s<+\infty$;

(iv) $\left(Y_{t}, U_{t}\right) \in \partial \phi, d P \times d s$, a.e. on $[0, T]$, in which

$$
\partial \phi(u)=\left\{u^{*} \in \mathbb{R}:\left\langle u^{*}, v-u\right\rangle+\phi(u) \leq \phi(v), \forall v \in \mathbb{R}\right\} .
$$


If the quadruple $(Y, Z, U, W)$ is the solution of $\operatorname{BSDE}(2.1)$, we use the symbol $(Y, Z, U$, $W) \in \operatorname{BSDE}(\xi, T ; \phi, f)$.

We propose some assumptions as follows.

(A1) The terminal value $\xi$ is $\mathcal{F}_{T}$-measurable such that

$$
\mathbb{E}|\xi|^{2}<\infty, \quad \xi \in \overline{\operatorname{Dom}(\phi)} \text { and } \mathbb{E}|\phi(\xi)|<+\infty
$$

(A2) The function $f: \Omega \times[0, T] \times I \times \mathbb{R} \times \mathbb{R}^{1 \times d} \times \mathbb{R}^{m} \rightarrow \mathbb{R}$ is progressively measurable, and for $i \in I$, it holds that

$$
\mathbb{E} \int_{0}^{T}|f(s, i, 0,0,0)|^{2} d s<+\infty, \quad \forall i \in I .
$$

(A3) For $i \in I, t \in[0, T], y, \tilde{y} \in \mathbb{R}, z, \tilde{z} \in \mathbb{R}^{1 \times d}$, there exist constants $\beta \in \mathbb{R}, \mu, \sigma, L \geq 0$, and $\varphi$ is an $\mathcal{F}_{t}$-progressively measurable process such that:

(a) $(y, z, w) \longmapsto f(t, i, y, z, w)$ is a continuous function.

(b) $\langle y-\tilde{y}, f(t, i, y, z, w)-f(t, i, \tilde{y}, z, w)\rangle \leq \beta|y-\tilde{y}|^{2}$, $|f(t, i, y, z, w)-f(t, i, y, \widetilde{z}, w)| \leq \mu|z-\widetilde{z}|,|f(t, i, y, z, w)-f(t, i, y, z, \widetilde{w})| \leq L|w-\widetilde{w}|$, $|f(t, i, y, 0,0)| \leq \varphi(t)+\sigma|y|$.

(c) $\mathbb{E}\left(\int_{0}^{T}|\varphi(s)|^{2} d s\right)<\infty$.

\section{A priori estimates and existence and uniqueness result}

\subsection{The results of existence and uniqueness}

Now, we begin by showing Theorem 2, which is the main results of this paper. But the proof of this theorem needs a lot of supporting propositions, so it will be presented later.

Theorem 2 Let assumptions (A1)-(A3) be satisfied. Then BSDE (2.1) has a unique solution $\left\{\left(Y_{t}, Z_{t}, U_{t}, W_{t}\right)\right\}_{0 \leq t \leq T}$ such that

$$
\begin{aligned}
& \mathbb{E} \int_{\tau}^{T}\left|Z_{s}\right|^{2} d s+\mathbb{E}\left(\sum_{j \in I} \int_{\tau}^{T}\left|W_{s}(j)\right|^{2} \mathbf{1}_{\left\{\alpha_{s-} \neq j\right\}} \lambda_{\alpha_{s-}, j}(s) d s\right) \leq C \Phi_{1}(\tau, T), \\
& \mathbb{E}\left(\sup _{\tau \leq t \leq T}\left|Y_{t}\right|^{2}\right) \leq C \Phi_{1}(\tau, T), \\
& \mathbb{E} \phi\left(Y_{\tau}\right) \leq C \Phi_{2}(\tau, T), \\
& \mathbb{E} \int_{\tau}^{T}\left|U_{s}\right|^{2} d s \leq C \Phi_{2}(\tau, T),
\end{aligned}
$$

where $\tau \in[0, T]$ is a stopping time,

$$
\begin{aligned}
& \Phi_{1}(\tau, T)=\mathbb{E}\left(|\xi|^{2}+\sum_{i \in I} \int_{\tau}^{T}|f(s, i, 0,0,0)|^{2} d s\right), \\
& \Phi_{2}(\tau, T)=\mathbb{E}\left(|\xi|^{2}+\phi(\xi)+\int_{\tau}^{T}|\varphi(s)|^{2} d s\right) .
\end{aligned}
$$


Proposition 3 Let assumptions (A1)-(A3) be satisfied. If $(Y, Z, U, W) \in \operatorname{BSDE}(\xi, T ; \phi, f)$ and $(\tilde{Y}, \tilde{Z}, \tilde{U}, \tilde{W}) \in \operatorname{BSDE}(\tilde{\xi}, T ; \phi, \tilde{f})$, we have

$$
\begin{aligned}
& \mathbb{E} \int_{0}^{T}\left|Z_{s}-\tilde{Z}_{s}\right|^{2} d s+\mathbb{E}\left(\sum_{j \in I} \int_{0}^{T}\left|W_{s}(j)-\tilde{W}_{s}(j)\right|^{2} \mathbf{1}_{\left\{\alpha_{s-\neq j\}}\right.} \lambda_{\alpha_{s-j} j}(s) d s\right) \leq C \Delta(T), \\
& \mathbb{E}\left(\sup _{0 \leq t \leq T}\left|Y_{t}-\tilde{Y}_{t}\right|^{2}\right) \leq C \Delta(T),
\end{aligned}
$$

where

$$
\Delta(T)=\mathbb{E}\left(|\xi-\tilde{\xi}|^{2}+\int_{0}^{T}\left|f\left(s, \alpha_{s}, Y_{s}, Z_{s}, W_{s}\right)-\tilde{f}\left(s, \alpha_{s}, Y_{s}, Z_{s}, W_{s}\right)\right|^{2} d s\right) .
$$

Corollary 4 Let assumptions (A1)-(A3) be satisfied. There exists a unique quadruple $(Y, Z, U, W)$ which satisfies BSDE (2.1) such that

$$
\begin{aligned}
& \lim _{t \rightarrow \infty} \mathbb{E}\left|Y_{t}\right|^{2}=0, \\
& \left(Y_{t}, U_{t}\right) \in \partial \phi, \quad d P \times d t, \quad \text { a.e. on }[0, T] .
\end{aligned}
$$

Moreover,

$$
\begin{aligned}
& \mathbb{E}\left(\sup _{t \geq 0}\left|Y_{t}\right|^{2}+\int_{0}^{\infty}\left|Z_{s}\right|^{2} d s+\sum_{j \in I} \int_{0}^{\infty}\left|W_{s}(j)\right|^{2} \mathbf{1}_{\left\{\alpha_{s-\neq j\}}\right.} \lambda_{\alpha_{s-j}}(s) d s\right) \\
& \leq C \mathbb{E} \sum_{i \in I} \int_{0}^{\infty}|f(s, i, 0,0,0)|^{2} d s, \\
& \sup _{t \geq 0} \mathbb{E} \phi\left(Y_{t}\right)+\mathbb{E} \int_{0}^{\infty}\left|U_{s}\right|^{2} d s \leq C \mathbb{E} \int_{0}^{\infty}|\varphi(s)|^{2} d s .
\end{aligned}
$$

\subsection{A priori estimates}

Before proving the previous results, we firstly give some a priori estimates on the solution. For $x \in \mathbb{R}$, we define a convex $C^{\prime}$-function $\phi_{\delta}, \delta>0$,

$$
\begin{aligned}
\phi_{\delta}(u) & =\inf \left\{\frac{1}{2}|u-v|^{2}+\delta \phi(v): v \in \mathbb{R}\right\} \\
& =\frac{1}{2}\left|u-J_{\delta} u\right|^{2}+\delta \phi\left(J_{\delta} u\right),
\end{aligned}
$$

where $J_{\delta} u=(I+\delta \partial \phi)^{-1}(u)$. Now we recall some properties of this approximation that appeared in [10]:

$$
\begin{aligned}
& \frac{1}{\delta} D \phi_{\delta}(u)=\frac{1}{\delta} \partial \phi_{\delta}(u)=\frac{1}{\delta}\left(u-J_{\delta} u\right) \in \partial \phi\left(J_{\delta} u\right), \\
& \left|J_{\delta} u-J_{\delta} v\right| \leq|u-v|, \quad \lim _{\delta \searrow_{\delta}} J_{\delta} u=\operatorname{Pr}_{\overline{\operatorname{Dom} \phi}}(u)
\end{aligned}
$$

for $u, v \in \mathbb{R}, \delta>0$. For the convexity of $\phi_{\delta}$, we have

$$
\phi_{\delta}(0) \geq \phi_{\delta}(u)+\left(D \phi_{\delta}(u),-u\right) .
$$


Hence, for $u \in \mathbb{R}$, it holds that

$$
0 \leq \phi_{\delta}(u) \leq\left(D \phi_{\delta}(u), u\right)
$$

By the monotonicity of $\partial \phi$ and (3.8a), we obtain

$$
\begin{aligned}
0 \leq & \left(\frac{1}{\delta} D \phi_{\delta}(u)-\frac{1}{\varepsilon} D \phi_{\varepsilon}(v), J_{\delta} u-J_{\varepsilon} v\right) \\
= & \left(\frac{1}{\delta} D \phi_{\delta}(u)-\frac{1}{\varepsilon} D \phi_{\varepsilon}(v), u-D \phi_{\delta}(u)-v+D \phi_{\varepsilon}(v)\right) \\
= & \left(\frac{1}{\delta} D \phi_{\delta}(u)-\frac{1}{\varepsilon} D \phi_{\varepsilon}(v), u-v\right)-\frac{1}{\delta}\left|D \phi_{\delta}(u)\right|^{2} \\
& -\frac{1}{\varepsilon}\left|D \phi_{\varepsilon}(v)\right|^{2}+\left(\frac{1}{\delta}+\frac{1}{\varepsilon}\right)\left(D \phi_{\delta}(u), D \phi_{\varepsilon}(v)\right) .
\end{aligned}
$$

Then, for $\delta, \varepsilon>0$, it holds that

$$
\left(\frac{1}{\delta} D \phi_{\delta}(u)-\frac{1}{\varepsilon} D \phi_{\varepsilon}(v), u-v\right) \geq-\left(\frac{1}{\delta}+\frac{1}{\varepsilon}\right)\left|D \phi_{\delta}(u)\right|\left|D \phi_{\varepsilon}(v)\right| .
$$

Now, we consider the approximating equation

$$
\begin{aligned}
Y_{t}^{\delta}= & \xi \int_{t}^{T} f\left(s, \alpha_{s}, Y_{s}^{\delta}, Z_{s}^{\delta}, W_{s}^{\delta}\right) d s-\frac{1}{\delta} \int_{t}^{T} D \phi_{\delta}\left(Y_{s}^{\delta}\right) d s \\
& -\int_{t}^{T} Z_{s}^{\delta} d B_{s}-\sum_{j \in I} \int_{t}^{T} W_{s}^{\delta}(j) d \tilde{\mathcal{V}}_{s}(j) .
\end{aligned}
$$

From Crépey and Moutoussi [11], $\left(Y^{\delta}, Z^{\delta}, U^{\delta}, W^{\delta}\right)$ is the unique solution of equation (3.10).

Proposition 5 Let assumptions (A1)-(A3) be satisfied and $\tau \in[0, T]$ be a stopping time. Then

$$
\begin{aligned}
& \mathbb{E}\left(\sup _{\tau \leq t \leq T}\left|Y_{t}^{\delta}\right|^{2}+\int_{\tau}^{T}\left|Z_{s}^{\delta}\right|^{2} d s+\sum_{j \in I} \int_{\tau}^{T}\left|W_{s}^{\delta}(j)\right|^{2} \mathbf{1}_{\left\{\alpha_{s-\neq j\}}\right.} \lambda_{\alpha_{s-j} j}(s) d s\right) \\
& \quad \leq C \Phi_{1}(\tau, T),
\end{aligned}
$$

where $\Phi_{1}$ is defined by (3.2a).

Proof Using Itô's formula for $\left|Y_{t}^{\delta}\right|^{2}$ yields that

$$
\begin{aligned}
&\left|Y_{t}^{\delta}\right|^{2}+\int_{t}^{T}\left|Z_{s}^{\delta}\right|^{2} d s+\sum_{j \in I} \int_{t}^{T}\left|W_{s}^{\delta}(j)\right|^{2} \mathbf{1}_{\left\{\alpha_{s-} \neq j\right\}} \lambda_{\alpha_{s-}, j}(s) d s \\
&+\frac{2}{\delta} \int_{t}^{T}\left(D \phi_{\delta}\left(Y_{s}^{\delta}\right), Y_{s}^{\delta}\right) d s \\
&=|\xi|^{2}+2 \int_{t}^{T}\left(f\left(s, \alpha_{s}, Y_{s}^{\delta}, Z_{s}^{\delta}, W_{s}^{\delta}\right), Y_{s}^{\delta}\right) d s
\end{aligned}
$$




$$
-2 \int_{t}^{T}\left(Y_{s}^{\delta}, Z_{s}^{\delta} d B_{s}\right)-2 \sum_{j \in I} \int_{t}^{T}\left(Y_{s}^{\delta}, W_{s}^{\delta}(j) d \tilde{V}_{s}(j)\right)
$$

Let us start with some terms in the equation above. On the one hand, according to the previous assumption, we have $\left(\frac{1}{\delta} D \phi_{\delta}\left(Y_{s}^{\delta}\right), Y_{s}^{\delta}\right) \geq 0$. On the other hand, from Schwarz's inequality, we get

$$
\begin{aligned}
& 2\left(f\left(s, \alpha_{s}, y, z, w\right), y\right) \\
& \leq 2 \beta|y|^{2}+2 \mu|y||z|+2 L|y||w|+2|y|\left|f\left(s, \alpha_{s}, 0,0,0\right)\right| \\
& \leq\left(2 \beta+(1+r) \mu^{2}+(1+r) L^{2}+r\right)|y|^{2} \\
&+\frac{1}{1+r}\left(|z|^{2}+|w|^{2}\right)+\frac{1}{r}\left|f\left(s, \alpha_{s}, 0,0,0\right)\right|^{2} .
\end{aligned}
$$

Hence,

$$
\begin{aligned}
\left|Y_{t}^{\delta}\right|^{2} & +\frac{r}{1+r} \int_{t}^{T}\left|Z_{s}^{\delta}\right|^{2} d s+\frac{r}{1+r} \sum_{j \in I} \int_{t}^{T}\left|W_{s}^{\delta}(j)\right|^{2} \mathbf{1}_{\left\{\alpha_{s-\neq j\}}\right.} \lambda_{\alpha_{s-j}, j}(s) d s \\
\leq & |\xi|^{2}+\left(2 \beta+\mu^{2}+L^{2}+\left(1+\mu^{2}+L^{2}\right) r\right) \int_{t}^{T}\left|Y_{s}^{\delta}\right|^{2} d s-2 \int_{t}^{T}\left(Y_{s}^{\delta}, Z_{s}^{\delta} d B_{s}\right) \\
& +\frac{1}{r} \int_{t}^{T}\left|f\left(s, \alpha_{s}, 0,0,0\right)\right|^{2} d s-2 \sum_{j \in I} \int_{t}^{T}\left(Y_{s}^{\delta}, W_{s}^{\delta}(j) d \tilde{V}_{s}(j)\right) .
\end{aligned}
$$

According to the main ideas of Proposition 2.1 in [12], we take the expectation in the above inequality. So

$$
\begin{gathered}
\mathbb{E}\left|Y_{t}^{\delta}\right|^{2}+\frac{r}{1+r}\left(\mathbb{E} \int_{t}^{T}\left|Z_{s}^{\delta}\right|^{2} d s+\mathbb{E} \sum_{j \in I} \int_{t}^{T}\left|W_{s}^{\delta}(j)\right|^{2} \mathbf{1}_{\left\{\alpha_{s-\neq j\}}\right\}} \lambda_{\alpha_{s-j}, j}(s) d s\right) \\
\leq \mathbb{E}\left(|\xi|^{2}+\frac{1}{r} \int_{t}^{T}\left|f\left(s, \alpha_{s}, 0,0,0\right)\right|^{2} d s\right)+\widetilde{C} \mathbb{E} \int_{t}^{T}\left|Y_{s}^{\delta}\right|^{2} d s,
\end{gathered}
$$

where $\widetilde{C}$ is a positive constant.

Then, by Gronwall's lemma, we get

$$
\mathbb{E}\left|Y_{t}^{\delta}\right|^{2} \leq \bar{C}
$$

where $\bar{C}$ is also a positive constant.

Thus, we have

$$
\mathbb{E}\left(\int_{0}^{T}\left|Z_{s}^{\delta}\right|^{2} d s+\sum_{j \in I} \int_{0}^{T}\left|W_{s}^{\delta}(j)\right|^{2} \mathbf{1}_{\left\{\alpha_{s-\neq f\}}\right.} \lambda_{\alpha_{s-j}}(s) d s\right) \leq C .
$$

In addition,

$$
\sup _{\tau \leq t \leq T}\left|Y_{t}^{\delta}\right|^{2} \leq|\xi|^{2}+\frac{1}{r} \sum_{i \in I} \int_{\tau}^{T}|f(s, i, 0,0,0)|^{2} d s
$$




$$
\begin{aligned}
& +2 \sup _{\tau \leq t \leq T}\left|\sum_{j \in I} \int_{t}^{T}\left(Y_{s}^{\delta}, W_{s}^{\delta}(j) d \tilde{V}_{s}(j)\right)\right| \\
& +2 \sup _{\tau \leq t \leq T}\left|\int_{t}^{T}\left(Y_{s}^{\delta}, Z_{s}^{\delta} d B_{s}\right)\right| .
\end{aligned}
$$

We obtain

$$
\begin{aligned}
& 2 \mathbb{E}\left(\sup _{\tau \leq t \leq T}\left|\int_{t}^{T}\left(Y_{s}^{\delta}, Z_{s}^{\delta} d B_{s}\right)\right|\right) \\
& \quad \leq \frac{1}{4} \mathbb{E}\left(\sup _{\tau \leq t \leq T}\left|Y_{t}^{\delta}\right|^{2}\right)+C_{1} \mathbb{E}\left(\int_{\tau}^{T}\left|Z_{s}^{\delta}\right|^{2} d s\right), \\
& 2 \mathbb{E}\left(\sup _{\tau \leq t \leq T}\left|\sum_{j \in I} \int_{t}^{T}\left(Y_{s}^{\delta}, W_{s}^{\delta}(j) d \tilde{V}_{s}(j)\right)\right|\right) \\
& \quad \leq \frac{1}{4} \mathbb{E}\left(\sup _{\tau \leq t \leq T}\left|Y_{t}^{\delta}\right|^{2}\right)+C_{2} \mathbb{E}\left(\sum_{j \in I} \int_{\tau}^{T}\left|W_{s}^{\delta}(j)\right|^{2} \mathbf{1}_{\left\{\alpha_{s-\neq j\}} \lambda_{\alpha_{s-j}}(s) d s\right) .}\right.
\end{aligned}
$$

Thus, we get

$$
\begin{aligned}
\mathbb{E}\left(\sup _{\tau \leq t \leq T}\left|Y_{t}^{\delta}\right|^{2}\right) \leq & \mathbb{E}\left(|\xi|^{2}+\frac{1}{r} \sum_{i \in I} \int_{\tau}^{T}|f(s, i, 0,0,0)|^{2} d s\right) \\
& +\frac{1}{2} \mathbb{E}\left(\sup _{\tau \leq t \leq T}\left|Y_{t}^{\delta}\right|^{2}\right)+C_{1} \mathbb{E}\left(\int_{\tau}^{T}\left|Z_{s}^{\delta}\right|^{2} d s\right) \\
& +C_{2} \mathbb{E}\left(\sum_{j \in I} \int_{\tau}^{T}\left|W_{s}^{\delta}(j)\right|^{2} \mathbf{1}_{\left\{\alpha_{s-\neq j\}} \lambda_{\alpha_{s-j}}(s) d s\right) .}\right.
\end{aligned}
$$

Proposition 6 Let assumptions (A1)-(A3) be satisfied. For $C>0$, we have

$$
\begin{aligned}
& \mathbb{E} \int_{\tau}^{T}\left(\frac{1}{\delta}\left|D \phi_{\delta}\left(Y_{s}^{\delta}\right)\right|\right)^{2} d s \leq C \Phi_{2}(\tau, T), \\
& \mathbb{E} \phi\left(J_{\delta} Y_{\tau}^{\delta}\right) \leq C \Phi_{2}(\tau, T), \\
& \mathbb{E}\left|Y_{\tau}^{\delta}-J_{\delta}\left(Y_{\tau}^{\delta}\right)\right|^{2} \leq \delta^{2} C \Phi_{2}(\tau, T),
\end{aligned}
$$

where $\Phi_{2}(\tau, T)$ is given by (3.2b), and $\tau \in[0, T]$ is a stopping time.

Proof Borrowing the ideas in Proposition 2.2 in [6], we just briefly show the result as follows.

The subdifferential inequality can be written as

$$
\phi_{\delta}\left(Y_{r}^{\delta}\right) \geq \phi_{\delta}\left(Y_{r^{\prime}}^{\delta}\right)+\left(D \phi_{\delta}\left(Y_{r^{\prime}}^{\delta}\right), Y_{r}^{\delta}-Y_{r^{\prime}}^{\delta}\right)
$$

for $r=t_{j+1} \wedge T, r^{\prime}=t_{j} \wedge T$, where $t=t_{0}<t_{1}<t_{2}<\cdots$, and $t_{j+1}-t_{j}=1 / n$. Summing up over $j$, and $n$ goes to $\infty$, we get

$$
\phi_{\delta}\left(Y_{t}^{\delta}\right)+\frac{1}{\delta} \int_{t}^{T}\left|D \phi_{\delta}\left(Y_{s}^{\delta}\right)\right|^{2} d s
$$




$$
\begin{aligned}
\leq & \phi_{\delta}(\xi)+\int_{t}^{T}\left(D \phi_{\delta}\left(Y_{s}^{\delta}\right), f\left(s, \alpha_{s}, Y_{s}^{\delta}, Z_{s}^{\delta}, W_{s}^{\delta}\right)\right) d s-\int_{t}^{T}\left(D \phi_{\delta}\left(Y_{s}^{\delta}\right), Z_{s}^{\delta} d B_{s}\right) \\
& -\sum_{j \in I} \int_{t}^{T}\left(D \phi_{\delta}\left(Y_{s}^{\delta}\right), W_{s}^{\delta}(j) d \tilde{V}_{s}(j)\right), \quad \forall t \geq 0, \text { a.s. }
\end{aligned}
$$

From (3.7), (3.8a), we get

$$
\begin{aligned}
& \frac{1}{2}\left|D \phi_{\delta}(y)\right|^{2}+\delta \phi\left(J_{\delta} y\right)=\phi_{\delta}(y), \quad \delta \phi\left(J_{\delta} y\right) \leq \phi_{\delta}(y), \\
& \phi_{\delta}(\xi) \leq \delta \phi(\xi), \quad y-J_{\delta} y=D \phi_{\delta}(y) .
\end{aligned}
$$

According to the previous assumption (A3), we have

$$
\begin{aligned}
& \left(D \phi_{\delta}(y), f(s, \alpha, y, z, w)\right) \\
& \quad \leq \frac{1}{2 \delta}\left|D \phi_{\delta}(y)\right|^{2}+\frac{\delta}{2}|f(s, \alpha, y, z, w)|^{2} \\
& \quad \leq \frac{1}{2 \delta}\left|D \phi_{\delta}(y)\right|^{2}+3 \delta\left(\mu^{2}|z|^{2}+L^{2}|w|^{2}+\sigma^{2}|y|^{2}+\varphi^{2}(t)\right) .
\end{aligned}
$$

The result follows.

Proposition 7 Let assumptions (A1)-(A3) be satisfied. For $\delta, \varepsilon>0$, we have

$$
\begin{aligned}
& \mathbb{E}\left(\int_{0}^{T}\left|Z_{s}^{\delta}-Z_{s}^{\varepsilon}\right|^{2} d s+\sum_{j \in I} \int_{0}^{T}\left|W_{s}^{\delta}(j)-W_{s}^{\varepsilon}(j)\right|^{2} \mathbf{1}_{\left\{\alpha_{s-} \neq j\right\}} \lambda_{\alpha_{s-}, j}(s) d s\right) \\
& \quad \leq(\delta+\varepsilon) C \Phi, \\
& \mathbb{E}\left(\sup _{0 \leq t \leq T}\left|Y_{t}^{\delta}-Y_{t}^{\varepsilon}\right|^{2}\right) \leq(\delta+\varepsilon) C \Phi,
\end{aligned}
$$

where

$$
\Phi=\mathbb{E}\left(|\xi|^{2}+\phi(\xi)+\sum_{i \in I} \int_{0}^{T}|f(s, i, 0,0,0)|^{2} d s\right)
$$

Proof By Itô's formula,we obtain

$$
\begin{aligned}
\mid Y_{t}^{\delta}- & \left.Y_{t}^{\varepsilon}\right|^{2}+\sum_{j \in I} \int_{t}^{T}\left|W_{s}^{\delta}(j)-W_{s}^{\varepsilon}(j)\right|^{2} \mathbf{1}_{\left\{\alpha_{s-} \neq j\right\}} \lambda_{\alpha_{s-}, j}(s) d s \\
& +\int_{t}^{T}\left|Z_{s}^{\delta}-Z_{s}^{\varepsilon}\right|^{2} d s+2 \int_{t}^{T}\left(Y_{s}^{\delta}-Y_{s}^{\varepsilon}, \frac{1}{\delta} D \phi_{\delta}\left(Y_{s}^{\delta}\right)-\frac{1}{\varepsilon} D \phi_{\varepsilon}\left(Y_{s}^{\varepsilon}\right)\right) d s \\
= & 2 \int_{t}^{T}\left(Y_{s}^{\delta}-Y_{s}^{\varepsilon}, f\left(s, \alpha_{s}, Y_{s}^{\delta}, Z_{s}^{\delta}, W_{s}^{\delta}\right)-f\left(s, \alpha_{s}, Y_{s}^{\varepsilon}, Z_{s}^{\varepsilon}, W_{s}^{\varepsilon}\right)\right) d s \\
& -2 \int_{t}^{T}\left(Y_{s}^{\delta}-Y_{s}^{\varepsilon},\left(Z_{s}^{\delta}-Z_{s}^{\varepsilon}\right) d B_{s}\right) \\
& -2 \sum_{j \in I} \int_{t}^{T}\left(Y_{s}^{\delta}-Y_{s}^{\varepsilon},\left(W_{s}^{\delta}(j)-W_{s}^{\varepsilon}(j)\right) d \tilde{V}_{s}(j)\right) .
\end{aligned}
$$


Moreover,

$$
\begin{aligned}
& 2\left(Y_{s}^{\delta}-Y_{s}^{\varepsilon}, f\left(s, \alpha_{s}, Y_{s}^{\delta}, Z_{s}^{\delta}, W_{s}^{\delta}\right)-f\left(s, \alpha_{s}, Y_{s}^{\varepsilon}, Z_{s}^{\varepsilon}, W_{s}^{\varepsilon}\right)\right) \\
& \quad \leq 2 \beta\left|Y_{s}^{\delta}-Y_{s}^{\varepsilon}\right|^{2}+2\left|Y_{s}^{\delta}-Y_{s}^{\varepsilon}\right| \mu\left|Z_{s}^{\delta}-Z_{s}^{\varepsilon}\right|+2\left|Y_{s}^{\delta}-Y_{s}^{\varepsilon}\right| L\left|W_{s}^{\delta}-W_{s}^{\varepsilon}\right| \\
& \quad \leq\left(2 \beta+\mu^{2}+L^{2}+\left(\mu^{2}+L^{2}\right) r\right)\left|Y_{s}^{\delta}-Y_{s}^{\varepsilon}\right|^{2}+\frac{1}{1+r}\left(\left|Z_{s}^{\delta}-Z_{s}^{\varepsilon}\right|^{2}+\left|W_{s}^{\delta}-W_{s}^{\varepsilon}\right|^{2}\right) .
\end{aligned}
$$

By (3.9), it holds that

$$
\begin{aligned}
(1- & \left.T\left(2 \beta+\mu^{2}+L^{2}+\left(\mu^{2}+L^{2}\right) r\right)\right) \sup _{t \leq s \leq T}\left|Y_{s}^{\delta}-Y_{s}^{\varepsilon}\right|^{2}+\frac{r}{1+r} \int_{t}^{T}\left|Z_{s}^{\delta}-Z_{s}^{\varepsilon}\right|^{2} d s \\
& +\frac{r}{1+r} \sum_{j \in I} \int_{t}^{T}\left|W_{s}^{\delta}(j)-W_{s}^{\varepsilon}(j)\right|^{2} \mathbf{1}_{\left\{\alpha_{s-} \neq j\right\}} \lambda_{\alpha_{s-}, j}(s) d s \\
\leq & 2\left(\frac{1}{\delta}+\frac{1}{\varepsilon}\right) \int_{t}^{T}\left|D \phi_{\delta}\left(Y_{s}^{\delta}\right)\right|\left|D \phi_{\varepsilon}\left(Y_{s}^{\varepsilon}\right)\right| d s-2 \int_{t}^{T}\left(Y_{s}^{\delta}-Y_{s}^{\varepsilon},\left(Z_{s}^{\delta}-Z_{s}^{\varepsilon}\right) d B_{s}\right) \\
& -2 \sum_{j \in I} \int_{t}^{T}\left(Y_{s}^{\delta}-Y_{s}^{\varepsilon},\left(W_{s}^{\delta}(j)-W_{s}^{\varepsilon}(j)\right) d \tilde{V}_{s}(j)\right) .
\end{aligned}
$$

From (3.12a), we get the following inequality, which shows the desired result, and $\Phi$ is given by (3.15).

$$
2\left(\frac{1}{\delta}+\frac{1}{\varepsilon}\right) \mathbb{E} \int_{t}^{T}\left|D \phi_{\delta}\left(Y_{s}^{\delta}\right)\right|\left|D \phi_{\varepsilon}\left(Y_{s}^{\varepsilon}\right)\right| d s \leq C(\delta+\varepsilon) \Phi
$$

\subsection{Proof of the results of existence and uniqueness}

With the a priori estimates in the previous section, the main purpose of this section is the proof of Theorem 2. Before that, we should start with the proof of Proposition 3.

Proof of Proposition 3 Using Itô's formula, we get

$$
\begin{aligned}
\mid Y_{t}- & \left.\tilde{Y}_{t}\right|^{2}+\int_{t}^{T}\left|Z_{s}-\tilde{Z}_{s}\right|^{2} d s+\sum_{j \in I} \int_{t}^{T}\left|W_{s}(j)-\tilde{W}_{s}(j)\right|^{2} \mathbf{1}_{\left\{\alpha_{s-} \neq j\right\}} \lambda_{\alpha_{s-}, j}(s) d s \\
& +2 \int_{t}^{T}\left(U_{s}-\tilde{U}_{s}, Y_{s}-\tilde{Y}_{s}\right) d s \\
= & |\xi-\tilde{\xi}|^{2}+2 \int_{t}^{T}\left(Y_{s}-\tilde{Y}_{s}, f\left(s, \alpha_{s}, Y_{s}, Z_{s}, W_{s}\right)-\tilde{f}\left(s, \alpha_{s}, \tilde{Y}_{s}, \tilde{Z}_{s}, \tilde{W}_{s}\right)\right) d s \\
& -2 \int_{t}^{T}\left(Y_{s}-\tilde{Y}_{s},\left(Z_{s}-\tilde{Z}_{s}\right) d B_{s}\right)-2 \sum_{j \in I} \int_{t}^{T}\left(Y_{s}-\tilde{Y}_{s},\left(W_{s}(j)-\tilde{W}_{s}(j)\right) d \tilde{V}_{s}(j)\right) .
\end{aligned}
$$

By the method similar to Proposition 5, we obtain

$$
\begin{aligned}
& 2\left(U_{s}-\tilde{U}_{s}, Y_{s}-\tilde{Y}_{s}\right) \geq 0, \quad d P \times d s \text { a.e., } \\
& 2\left(Y_{s}-\tilde{Y}_{s}, f\left(s, \alpha_{s}, Y_{s}, Z_{s}, W_{s}\right)-\tilde{f}\left(s, \alpha_{s}, \tilde{Y}_{s}, \tilde{Z}_{s}, \tilde{W}_{s}\right)\right) \\
& \quad \leq 2\left(Y_{s}-\tilde{Y}_{s}, f\left(s, \alpha_{s}, Y_{s}, Z_{s}, W_{s}\right)-\tilde{f}\left(s, \alpha_{s}, Y_{s}, Z_{s}, W_{s}\right)\right)
\end{aligned}
$$




$$
\begin{aligned}
& +2\left|Y_{s}-\tilde{Y}_{s}\right| \tilde{L}\left|W_{s}-\tilde{W}_{s}\right|+2\left|Y_{s}-\tilde{Y}_{s}\right| \tilde{\mu}\left|Z_{s}-\tilde{Z}_{s}\right|+2 \tilde{\beta}\left|Y_{s}-\tilde{Y}_{s}\right|^{2} \\
= & \left(2 \tilde{\beta}+\tilde{L}^{2}+\tilde{\mu}^{2}+\left(1+\tilde{L}^{2}+\tilde{\mu}^{2}\right) r\right)\left|Y_{s}-\tilde{Y}_{s}\right|^{2}+\frac{1}{1+r}\left(\left|Z_{s}-\tilde{Z}_{s}\right|^{2}+\left|W_{s}-\tilde{W}_{s}\right|^{2}\right) \\
& +\frac{1}{r}\left|f\left(s, \alpha_{s}, Y_{s}, Z_{s}, W_{s}\right)-\tilde{f}\left(s, \alpha_{s}, Y_{s}, Z_{s}, W_{s}\right)\right|^{2},
\end{aligned}
$$

where $\beta, \mu, L$ are replaced by $\tilde{\beta}, \tilde{\mu}, \tilde{L}$. Taking the expectation and using Gronwall's lemma, we have (3.3a) and (3.3b).

Proof of Theorem 2 Uniqueness can be obtained simply by Proposition 3. The existence of the solution $(Y, Z, U, W)$ can be drawn from the limit of the quadruple $\left(Y_{s}^{\delta}, Z_{s}^{\delta}, \frac{1}{\delta} D \phi_{\delta}\left(Y_{s}^{\delta}\right), W_{s}^{\delta}\right)$.

From Proposition 7, we have

$$
\lim _{\delta \searrow 0} Y^{\delta}=Y, \quad \lim _{\delta \searrow 0} Z^{\delta}=Z, \quad \lim _{\delta \searrow 0} W^{\delta}=W
$$

Passing to the limit in (3.11), we can get (3.1a) and (3.1b). From (3.12a) and (3.12c), we have

$$
\lim _{\delta \searrow 0} J_{\delta}\left(Y^{\delta}\right)=Y, \quad \lim _{\delta \searrow 0} \mathbb{E}\left(\left|J_{\delta}\left(Y_{\tau}^{\delta}\right)-Y_{\tau}\right|^{2}\right)=0
$$

in which $\tau \in[0, T]$ is a stopping time.

Because of (3.12b), (3.14b), we get (3.1c) and (iii). For each $\delta>0$, define $U_{t}^{\delta}=\frac{1}{\delta} D \phi_{\delta}\left(Y_{t}^{\delta}\right)$ and $\bar{U}_{t}^{\delta}=\int_{0}^{t} U_{s}^{\delta} d s$. Consider (3.10) and convergence results, there exists a progressively measurable process $\left\{\bar{U}_{t}, 0 \leq t \leq T\right\}$ such that

$$
\mathbb{E}\left(\sup _{0 \leq t \leq T}\left|\bar{U}_{t}^{\delta}-\bar{U}_{t}\right|^{2}\right) \rightarrow 0, \quad \delta \rightarrow 0
$$

Moreover, from (3.12a), we obtain $\sup _{\delta>0} \mathbb{E} \int_{0}^{T}\left|U_{t}^{\delta}\right|^{2} d t<\infty$. Then we get (3.1d).

$$
\text { For } 0 \leq a<b \leq T,\left[\mathbb{E} \int_{0}^{T}|V|^{2} d s\right]^{1 / 2}<\infty \text {, }
$$

$$
\int_{a}^{b}\left(U_{t}^{\delta}, V_{t}-Y_{t}^{\delta}\right) d t \rightarrow \int_{a}^{b}\left(U_{t}, V_{t}-Y_{t}\right) d t
$$

From equation (3.12a), we have $\int_{a}^{b}\left(U_{t}^{\delta}, J_{\delta}\left(Y_{t}^{\delta}\right)-Y_{t}^{\delta}\right) d t \rightarrow 0$.

Since $U_{t}^{\delta} \in \partial \phi\left(U_{\delta}\left(Y_{t}^{\delta}\right)\right)$,

$$
\int_{a}^{b}\left(U_{t}^{\delta}, V_{t}-J_{\delta}\left(Y_{t}^{\delta}\right)\right) d t+\int_{a}^{b} \phi\left(J_{\delta}\left(Y_{t}^{\delta}\right)\right) d t \leq \int_{a}^{b} \phi\left(V_{t}\right) d t .
$$

Then we get

$$
\int_{a}^{b}\left(U_{t}, V_{t}-Y_{t}\right) d t+\int_{a}^{b} \phi\left(Y_{t}\right) d t \leq \int_{a}^{b} \phi\left(V_{t}\right) d t .
$$

The proof of Theorem 2 has been completed. 
Proof of Corollary 4 Let $\left(Y^{n}, Z^{n}, U^{n}, W^{n}\right) \in \operatorname{BSDE}(0, n ; \phi, f)$ for each $n \geq 1$. According to (3.1a)-(3.1d) in Theorem 2, we have

$$
\begin{aligned}
& \mathbb{E}\left(\int_{0}^{n}\left|Z_{s}^{n}\right|^{2} d s+\sum_{j \in I} \int_{0}^{n}\left|W_{s}^{n}(j)\right|^{2} \mathbf{1}_{\left\{\alpha_{s-\neq \neq j}\right.} \lambda_{\alpha_{s-j}}(s) d s\right) \\
& \quad \leq C_{1} \mathbb{E}\left(\sum_{i \in I} \int_{0}^{\infty}|f(s, i, 0,0,0)|^{2} d s\right), \\
& \mathbb{E}\left(\sup _{0 \leq s \leq n}\left|Y_{s}^{n}\right|^{2}\right) \leq C_{1} \mathbb{E}\left(\sum_{i \in I} \int_{0}^{\infty}|f(s, i, 0,0,0)|^{2} d s\right), \\
& \mathbb{E} \phi\left(Y_{t}^{n}\right) \leq C_{2} \mathbb{E} \int_{0}^{\infty}|\varphi(s)|^{2} d s, \\
& \mathbb{E} \int_{0}^{n}\left|U_{s}^{n}\right|^{2} d s \leq C_{2} \mathbb{E} \int_{0}^{\infty}|\varphi(s)|^{2} d s,
\end{aligned}
$$

and $Y_{s}^{n}=Y_{n}^{n}=0, Z_{s}^{n}=0, U_{s}^{n}=0, W_{s}^{n}=0$ for $s>n$.

Let $m>n$, then we get

$$
\begin{aligned}
Y_{t}^{m}= & Y_{n}^{m}+\int_{t}^{n} f\left(s, \alpha_{s}, Y_{s}^{m}, Z_{s}^{m}, W_{s}^{m}\right) d s-\int_{t}^{n} U_{s}^{m} d s \\
& -\int_{t}^{n} Z_{s}^{m} d B_{s}-\sum_{j \in I} \int_{t}^{n} W_{s}^{m}(j) d \tilde{\mathcal{V}}_{s}(j)
\end{aligned}
$$

for $t \in[0, n]$. From Proposition 3, we have

$$
\begin{aligned}
& \mathbb{E}\left(\int_{0}^{n}\left|Z_{s}^{n}-Z_{s}^{m}\right|^{2} d s+\sum_{j \in I} \int_{0}^{n}\left|W_{s}^{n}(j)-W_{s}^{m}(j)\right|^{2} \mathbf{1}_{\left\{\alpha_{s-\neq j\}}\right.} \lambda_{\alpha_{s-j} j}(s) d s\right) \\
& \quad \leq C \mathbb{E}\left|Y_{n}^{m}\right|^{2}, \\
& \mathbb{E}\left(\sup _{0 \leq s \leq n}\left|Y_{s}^{n}-Y_{s}^{m}\right|^{2}\right) \leq C \mathbb{E}\left|Y_{n}^{m}\right|^{2} .
\end{aligned}
$$

From (3.1b), we obtain

$$
\mathbb{E}\left|Y_{T}^{m}\right|^{2} \leq \mathbb{E}\left(\sup _{T \leq t \leq m}\left|Y_{t}^{m}\right|^{2}\right) \leq C \mathbb{E}\left(\sum_{i \in I} \int_{T}^{\infty}|f(s, i, 0,0,0)|^{2} d s\right) \rightarrow 0, \quad T=n \rightarrow \infty .
$$

There exists $(Y, Z, U, W)$ satisfying (i) for all $T>0$, as $n \rightarrow \infty$, we obtain

$$
Y^{n} \rightarrow Y, \quad \mathbb{E}\left|Y_{T}\right|^{2} \leq C \mathbb{E}\left(\sum_{i \in I} \int_{T}^{\infty}|f(s, i, 0,0,0)|^{2} d s\right), \quad Z^{n} \rightarrow Z, \quad \bar{U}^{n} \rightarrow \bar{U}
$$

where $\bar{U}_{t}^{n}=\int_{0}^{t} U_{s}^{n} d s$, and $\bar{U}$ is absolutely continuous. $(Y, Z, U, W)$ satisfies Corollary 4 , in which $U=d \bar{U} / d t$.

If $(Y, Z, U, W)$ and $\left(Y^{\prime}, Z^{\prime}, U^{\prime}, W^{\prime}\right)$ are two solutions of BSDE (2.1) satisfying (3.5a) and (3.5b), then

$$
\mathbb{E}\left(\sup _{0 \leq s \leq n}\left|Y_{s}-Y_{s}^{\prime}\right|^{2}\right)
$$




$$
\begin{aligned}
& +\mathbb{E}\left(\int_{0}^{n}\left|Z_{s}-Z_{s}^{\prime}\right|^{2} d s+\sum_{j \in I} \int_{0}^{n}\left|W_{s}(j)-W_{s}^{\prime}(j)\right|^{2} \mathbf{1}_{\left\{\alpha_{s-\neq j\}}\right.} \lambda_{\alpha_{s-j}}(s) d s\right) \\
\leq & C_{1} \mathbb{E}\left|Y_{n}-Y_{n}^{\prime}\right|^{2},
\end{aligned}
$$

we get $Y=Y^{\prime}, Z=Z^{\prime}, W=W^{\prime}$ for $n \rightarrow \infty$; $U$ is uniquely defined by BSDE (2.1).

\section{Weak convergence of multi-valued BSDEs with Markov switching}

\subsection{Asymptotic property of SDE with the singularly perturbed Markov chain}

Let $\alpha^{\varepsilon}(t)$ be a Markov chain governed by $Q^{\varepsilon}(t)=\left(\lambda_{i j}^{\varepsilon}(t)\right)$ that satisfies

$$
Q^{\varepsilon}(t)=\frac{1}{\varepsilon} \widetilde{Q}(t)+\widehat{Q}(t), \quad t \geq 0,
$$

$\widetilde{Q}(t)$ represents the fast part and $\widehat{Q}(t)$ represents the slow part. More details on singularly perturbed Markov chains can be found in Tao et al. [3]. The next lemma can be found in [13].

Lemma 8 Define the aggregated process $\bar{\alpha}^{\varepsilon}=\left\{\bar{\alpha}_{t}^{\varepsilon} ; 0 \leq t \leq T\right\}$ as follows: $\forall k \in\{1, \ldots, l\}$, $\bar{\alpha}_{t}^{\varepsilon}=k$, when $\alpha_{t}^{\varepsilon} \in I_{k}$. Then, as $\varepsilon \rightarrow 0$, $\bar{\alpha}^{\varepsilon}$ converges weakly to a continuous-time Markov chain $\bar{\alpha}$ with the generator

$$
\bar{Q}(t)=\operatorname{diag}\left(v^{1}(t), \ldots, v^{l}(t)\right) \widehat{Q}(t) \operatorname{diag}\left(\mathbb{I}_{m_{1}}, \ldots, \mathbb{I}_{m_{l}}\right) .
$$

Here, $\forall k \in\{1, \ldots, l\}, v^{k}$ is the quasi-stationary distribution of $\widetilde{Q}^{k}$ and $\mathbb{I}_{m_{k}}=\{1, \ldots, 1\}^{*} \in$ $\mathbb{R}^{m_{k}}$. Here, ${ }^{*}$ denotes the transpose.

Now, we present a diffusion process $X_{t}^{\varepsilon}$ as follows:

$$
X_{t}^{\varepsilon}=x+\int_{0}^{t} b\left(s, \alpha_{s}^{\varepsilon}, X_{s}^{\varepsilon}\right) d s+\int_{0}^{t} \sigma\left(s, \alpha_{s}^{\varepsilon}, X_{s}^{\varepsilon}\right) d B_{s}, \quad 0 \leq t \leq T,
$$

there exist $p, q \geq 0$ such that

$$
\sup _{\varepsilon} \mathbb{E}\left(\left|X_{t}^{\varepsilon}\right|^{2 p}+\int_{0}^{t}\left|X_{s}^{\varepsilon}\right|^{2 q} d s\right)<\infty
$$

In the diffusion process above, we present the conditions of $b$ and $\sigma$ : for any $i \in I, b(\cdot, i, \cdot)$ and $\sigma(\cdot, i, \cdot)$ are measurable,

$$
\left|b(t, i, x)-b\left(t, i, x^{*}\right)\right| \leq C^{\prime}\left|x-x^{*}\right|, \quad|b(t, i, 0)| \leq C^{\prime},
$$

and

$$
\left|\sigma(t, i, x)-\sigma\left(t, i, x^{*}\right)\right| \leq C^{\prime}\left|x-x^{*}\right|, \quad|\sigma(t, i, 0)| \leq C^{\prime} .
$$

More details can also be found in [3]. 
Let $a=\sigma \sigma^{*}$. We define

$$
\mathcal{D}^{\varepsilon} u(t, i, x)=\left(\frac{\partial}{\partial t}+\mathcal{L}\right) u(t, i, x)+f(t, i, x, u(t, i, x))+\sum_{j \neq i} \lambda_{i j}^{\varepsilon}(t)[u(t, j, x)-u(t, i, x)],
$$

where

$$
\mathcal{L} u(t, i, x)=\frac{1}{2} \sum_{p, q=1}^{n} a_{p q}(t, i, x) \frac{\partial^{2}}{\partial x_{p} \partial x_{q}} u(t, i, x)+\sum_{p=1}^{n} b_{p}(t, i, x) \frac{\partial}{\partial x_{p}} u(t, i, x) .
$$

Now, we propose the following asymptotic property for the above generators.

Lemma 9 Assume that $\bar{\alpha}^{\varepsilon}(t)$ is a Markov chain and $b(t, i, x), \sigma(t, i, x)$ satisfy the above conditions. Then $\left(X^{\varepsilon}(\cdot), \bar{\alpha}^{\varepsilon}(\cdot)\right)$ converges weakly under the Skorohod topology to a process $(\bar{X}(\cdot), \bar{\alpha}(\cdot))$. Moreover, $(\bar{X}(\cdot), \bar{\alpha}(\cdot))$ is a solution of the martingale problem with operator

$$
\overline{\mathcal{D}} u(t, i, x)=\left(\frac{\partial}{\partial t}+\overline{\mathcal{L}}\right) u(t, i, x)+\bar{f}(t, i, x, u(t, i, x))+\sum_{j \neq i} \bar{\lambda}_{i j}(t)[u(t, j, x)-u(t, i, x)],
$$

where

$$
\begin{aligned}
& \overline{\mathcal{L}} u(t, i, x)=\frac{1}{2} \sum_{p, q=1}^{n} \bar{a}_{p q}(t, i, x) \frac{\partial^{2}}{\partial x_{p} \partial x_{q}} u(t, i, x)+\sum_{p=1}^{n} \bar{b}_{p}(t, i, x) \frac{\partial}{\partial x_{p}} u(t, i, x), \\
& \bar{a}(t, i, x)=\sum_{j=1}^{m_{i}} v_{j}^{i}(t) a\left(t, s_{i j}, x\right), \quad \bar{b}(t, i, x)=\sum_{j=1}^{m_{i}} v_{j}^{i}(t) b\left(t, s_{i j}, x\right), \\
& \bar{f}(t, i, x, u)=\sum_{j=1}^{m_{i}} v_{j}^{i}(t) f\left(t, s_{i j}, x, u\right) .
\end{aligned}
$$

\subsection{Weak convergence of multi-valued BSDEs with Markov switching}

Let us consider the following assumptions:

(A4) $g: \mathbb{R}^{d} \rightarrow \mathbb{R}$ and $f:[0, T] \times I \times \mathbb{R}^{d} \times \mathbb{R} \rightarrow \mathbb{R}$ are all continuous.

(A5) $|g(x)| \leq C\left(1+|x|^{p}\right)$.

(A6) $|f(t, i, x, y)| \leq C\left(1+|x|^{q}+|y|^{r}\right)$.

(A7) $\langle f(t, i, x, y)-f(t, i, x, \widetilde{y}), y-\widetilde{y}\rangle \leq \beta|y-\widetilde{y}|^{2}$.

(A8) $\int_{0}^{T}|f(t, i, x, 0)|^{2} d t<\infty, \forall i \in I$.

Let $\left\{Y_{t}^{\varepsilon}, Z_{t}^{\varepsilon}, U_{t}^{\varepsilon}, W_{t}^{\varepsilon} ; 0 \leq t \leq T\right\}$ be the unique solution of the following BSDE with $\alpha_{t}=\alpha_{t}^{\varepsilon}$ :

$$
\begin{aligned}
Y_{t}^{\varepsilon}= & g\left(X_{T}^{\varepsilon}\right)+\int_{t}^{T} f\left(s, \alpha_{s}^{\varepsilon}, X_{s}^{\varepsilon}, Y_{s}^{\varepsilon}\right) d s-\int_{t}^{T} Z_{s}^{\varepsilon} d B_{s} \\
& -\sum_{j \in I} \int_{t}^{T} W_{s}^{\varepsilon}(j) d \tilde{\mathcal{V}}_{s}^{\varepsilon}(j)-\int_{t}^{T} U_{s}^{\varepsilon} d s .
\end{aligned}
$$

Next, we prove that the processes $\left(Y^{\varepsilon}, Z^{\varepsilon}, U^{\varepsilon}, W^{\varepsilon}\right)$ converge in law to $(Y, Z, U, W)$, which is the unique solution of the following BSDE:

$$
Y_{t}=g\left(X_{T}\right)+\int_{t}^{T} f\left(s, \alpha_{s}, X_{s}, Y_{s}\right) d s-\int_{t}^{T} Z_{s} d B_{s}
$$




$$
-\sum_{j \in I} \int_{t}^{T} W_{s}(j) d \tilde{\mathcal{V}}_{s}(j)-\int_{t}^{T} U_{s} d s
$$

Theorem 10 ([14]) The sequence of quasi-martingale $\left\{\rho_{t}^{n} ; 0 \leq t \leq T\right\}$ defined on the filtrated probability space $\left(\Omega,\left\{\mathcal{F}_{t}\right\}_{0 \leq t \leq T}, \mathbb{P}\right)$ is tight if

$$
\sup _{n}\left(\sup _{0 \leq t \leq T} E\left|\rho_{t}^{n}\right|+C V_{T}\left(\rho^{n}\right)\right)<\infty
$$

where

$$
C V_{T}\left(\rho^{n}\right)=\sup \mathbb{E}\left(\sum_{i}\left|\mathbb{E}\left[\rho_{t_{i+1}}^{n}-\rho_{t_{i}}^{n} \mid \mathcal{F}_{t_{i}}\right]\right|\right)
$$

with the supremum taken over all partitions of the interval $[0, T]$.

In what follows, let

$$
M_{t}^{\varepsilon}=\int_{0}^{t} Z_{s}^{\varepsilon} d B_{s}+\sum_{j \in I} \int_{0}^{t} W_{s}^{\varepsilon}(j) d \tilde{\mathcal{V}}_{s}^{\varepsilon}(j), \quad M_{t}=\int_{0}^{t} Z_{s} d B_{s}+\sum_{j \in \bar{I}} \int_{0}^{t} W_{s}(j) d \tilde{\mathcal{V}}_{s}(j) .
$$

Now, we introduce the first result of this section.

Theorem 11 Under the assumptions stated above, the sequence of processes $\left(X^{\varepsilon}, Y^{\varepsilon}, M^{\varepsilon}\right.$, $\left.U^{\varepsilon}\right)$ converges in law to $(X, Y, M, U)$ as $\varepsilon$ goes to zero.

Next, we give Lemma 12 that appeared in Billingsley [15] to help us complete the proof of Theorem 11.

Lemma 12 Let $U^{\varepsilon}$ be a sequence of random variables defined on the same probability spaces. For any $\varepsilon \geq 0$, we assume that there exists a sequence of random variables $\left(U^{\varepsilon, n}\right)_{n}$ such that

- $U^{\varepsilon, n} \stackrel{\text { dist }}{\Longrightarrow} U^{0, n}$ as $\varepsilon$ goes to zero.

- $U^{\varepsilon, n} \Longrightarrow U^{\varepsilon}$ as $n \rightarrow+\infty$, uniformly in $\varepsilon$.

- $U^{0, n} \Longrightarrow U^{0}$ as $n \rightarrow+\infty$.

Then $U^{\varepsilon}$ converge in distribution to $U^{0}$.

For $n \geq 1$, we consider the penalized forms of (4.1) and (4.2):

$$
\begin{aligned}
Y_{t}^{\varepsilon, n}= & g\left(X_{T}^{\varepsilon}\right)+\int_{t}^{T} f\left(s, \alpha_{s}^{\varepsilon}, X_{s}^{\varepsilon}, Y_{s}^{\varepsilon, n}\right) d s-\int_{t}^{T} Z_{s}^{\varepsilon, n} d B_{s} \\
& -\sum_{j \in I} \int_{t}^{T} W_{s}^{\varepsilon, n}(j) d \tilde{\mathcal{V}}_{s}^{\varepsilon}(j)-\int_{t}^{T} U_{s}^{\varepsilon, n} d s
\end{aligned}
$$

and

$$
Y_{t}^{n}=g\left(X_{T}\right)+\int_{t}^{T} f\left(s, \alpha_{s}, X_{s}, Y_{s}^{n}\right) d s-\int_{t}^{T} Z_{s}^{n} d B_{s}
$$




$$
-\sum_{j \in I} \int_{t}^{T} W_{s}^{n}(j) d \tilde{\mathcal{V}}_{s}(j)-\int_{t}^{T} U_{s}^{0, n} d s
$$

Let $M_{t}^{\varepsilon, n}=\int_{0}^{t} Z_{s}^{\varepsilon, n} d B_{s}+\sum_{j \in I} \int_{0}^{t} W_{s}^{\varepsilon, n}(j) d \tilde{\mathcal{V}}_{s}^{\varepsilon}(j)$. With the preparation above, we can now prove Theorem 11 step by step.

Lemma 13 Under the assumptions of Theorem 11 , for $n \geq 1,\left(Y^{\varepsilon, n}, M^{\varepsilon, n}\right)$ converges in law to $\left(Y^{n}, M^{n}\right)$.

Proof We shall prove this result in the following four steps.

Step 1. A Priori Estimates. By the standard arguments, we have

$$
\sup _{\varepsilon} \mathbb{E}\left(\sup _{0 \leq s \leq t}\left|Y_{s}^{\varepsilon, n}\right|^{2}+\left\langle M^{\varepsilon, n}\right\rangle_{t}+\frac{2 \gamma}{\varepsilon} \int_{s}^{t}\left|D \phi_{\varepsilon}\left(Y_{r}^{\varepsilon, n}\right)\right| d r\right)<+\infty
$$

where

$$
\left\langle M^{\varepsilon, n}\right\rangle_{t}=\int_{0}^{t}\left|Z_{s}^{\varepsilon, n}\right|^{2} d s+\sum_{j \in I} \int_{0}^{t}\left|W_{s}^{\varepsilon, n}(j)\right|^{2} \mathbf{1}_{\left\{\alpha_{s-}^{\varepsilon} \neq j\right]} \lambda_{\alpha_{s-j}}^{\varepsilon}(s) d s .
$$

Step 2. Tightness. We have

$$
C V_{T}\left(Y^{\varepsilon, n}\right) \leq \int_{0}^{T}\left|f\left(s, \alpha_{s}^{\varepsilon}, X_{s}^{\varepsilon}, Y_{s}^{\varepsilon, n}\right)\right| d s+\frac{1}{\varepsilon} \int_{0}^{T}\left|D \phi_{\varepsilon}\left(Y_{s}^{\varepsilon, n}\right)\right| d s .
$$

Then we obtain

$$
\sup _{\varepsilon}\left[C V_{T}\left(Y^{\varepsilon, n}\right)+\mathbb{E}\left(\sup _{0 \leq s \leq t}\left|Y_{s}^{\varepsilon, n}\right|^{2}\right)+\sup _{0 \leq s \leq t} \mathbb{E}\left|M_{s}^{\varepsilon, n}\right|\right]<+\infty .
$$

Hence, the sequence $\left\{\left(Y_{s}^{\varepsilon, n}, M_{s}^{\varepsilon, n}\right) ; 0 \leq s \leq t\right\}$ satisfies the Meyer-Zheng tightness criterion. There exists a subsequence $\left(Y^{\varepsilon, n}, M^{\varepsilon, n}\right) \Longrightarrow\left(Y^{n}, M^{n}\right)$.

Step 3. Convergence in Law. We first derive the limit process of $\int_{0}^{t} f\left(s, \alpha_{s}^{\varepsilon}, X_{s}^{\varepsilon}, Y_{s}^{\varepsilon, n}\right) d s$. For the state space of $\alpha^{\varepsilon}(t)$ that is $I=\left\{s_{11}, \ldots, s_{1 m_{1}}, \ldots, s_{l 1}, \ldots, s_{l m_{l}}\right\}$, we have

$$
\begin{aligned}
& \int_{0}^{t} f\left(s, \alpha_{s}^{\varepsilon}, X_{s}^{\varepsilon}, Y_{s}^{\varepsilon, n}\right) d s \\
& =\sum_{i=1}^{l} \sum_{j=1}^{m_{i}} \int_{0}^{t} f\left(s, s_{i j}, X_{s}^{\varepsilon}, Y_{s}^{\varepsilon, n}\right) \mathbf{1}_{\left\{\alpha_{s}^{\varepsilon}=s_{i j}\right\}} d s \\
& =\sum_{i=1}^{l} \sum_{j=1}^{m_{i}} \int_{0}^{t} f\left(s, s_{i j}, X_{s}^{\varepsilon}, Y_{s}^{\varepsilon, n}\right) v_{j}^{i}(s) \mathbf{1}_{\left\{\bar{\alpha}_{s}^{\varepsilon}=i\right\}} d s \\
& \quad+\sum_{i=1}^{l} \sum_{j=1}^{m_{i}} \int_{0}^{t} f\left(s, s_{i j}, X_{s}^{\varepsilon}, Y_{s}^{\varepsilon, n}\right)\left(\mathbf{1}_{\left\{\alpha_{s}^{\varepsilon}=s_{i j}\right\}}-v_{j}^{i}(s) \mathbf{1}_{\left\{\bar{\alpha}_{s}^{\varepsilon}=i\right\}}\right) d s .
\end{aligned}
$$

By Yin and Zhang [13], for $i=1,2, \ldots, l$ and $j=1, \ldots, m_{i}$, as $\varepsilon \rightarrow 0$, it follows that

$$
\sup _{0 \leq t \leq T} \mathbb{E}\left(\int_{0}^{t}\left(\mathbf{1}_{\left\{\alpha_{s}^{\varepsilon}=s_{i j}\right\}}-v_{j}^{i}(s) \mathbf{1}_{\left\{\bar{\alpha}_{s}^{\varepsilon}=i\right\}}\right) d s\right)^{2} \rightarrow 0 .
$$


So we get

$$
\begin{aligned}
& \sup _{0 \leq t \leq T} \mathbb{E}\left|\int_{0}^{t} f\left(s, s_{i j}, X_{s}^{\varepsilon}, Y_{s}^{\varepsilon, n}\right)\left(\mathbf{1}_{\left\{\alpha_{s}^{\varepsilon}=s_{i j}\right\}}-v_{j}^{i}(s) \mathbf{1}_{\left\{\bar{\alpha}_{s}^{\varepsilon}=i\right\}}\right) d s\right| \\
& \quad \leq \sup _{0 \leq t \leq T} \mathbb{E}\left|\sup _{0 \leq s \leq t}\right| f\left(s, s_{i j}, X_{s}^{\varepsilon}, Y_{s}^{\varepsilon, n}\right)\left|\int_{0}^{t}\left(\mathbf{1}_{\left\{\alpha_{s}^{\varepsilon}=s_{i j}\right\}}-v_{j}^{i}(s) \mathbf{1}_{\left\{\bar{\alpha}_{s}^{\varepsilon}=i\right\}}\right) d s\right| \\
& \quad \leq \sup _{0 \leq t \leq T}\left(\mathbb{E}_{0 \leq s \leq t}\left|f\left(s, s_{i j}, X_{s}^{\varepsilon}, Y_{s}^{\varepsilon, n}\right)\right|^{2}\right)^{\frac{1}{2}}\left[\mathbb{E}\left(\int_{0}^{t}\left(\mathbf{1}_{\left\{\alpha_{s}^{\varepsilon}=s_{i j}\right\}}-v_{j}^{i}(s) \mathbf{1}_{\left\{\bar{\alpha}_{s}^{\varepsilon}=i\right\}}\right) d s\right)^{2}\right]^{1 / 2} \\
& \quad \leq C \sup _{0 \leq t \leq T}\left[\mathbb{E}\left(\int_{0}^{t}\left(\mathbf{1}_{\left\{\alpha_{s}^{\varepsilon}=s_{i j}\right\}}-v_{j}^{i}(s) \mathbf{1}_{\left\{\bar{\alpha}_{s}^{\varepsilon}=i\right\}}\right) d s\right)^{2}\right]^{1 / 2} \rightarrow 0 .
\end{aligned}
$$

Following the inequality above, we have

$$
\sum_{i=1}^{l} \sum_{j=1}^{m_{i}} \int_{0}^{t} f\left(s, s_{i j}, X_{s}, Y_{s}^{n}\right) v_{j}^{i}(s) \mathbf{1}_{\left\{\bar{\alpha}_{s}=i\right\}} d s=\int_{t}^{T} f\left(s, \bar{\alpha}_{s}, X_{s}, Y_{s}^{n}\right) d s .
$$

In (4.3), let $\varepsilon \rightarrow 0$,

$$
Y_{t}^{n}=g\left(X_{T}\right)+\int_{t}^{T} f\left(s, \bar{\alpha}_{s}, X_{s}, Y_{s}^{n}\right) d s-M_{T}^{n}+M_{t}^{n}-\frac{1}{\varepsilon} \int_{t}^{T} D \phi_{\varepsilon}\left(Y_{s}^{n}\right) d s .
$$

For $f(t, j, x), j \in \bar{I}$, we define

$$
\tilde{f}(t, i, x)=\sum_{j=1}^{l} f(t, j, x) I_{\left\{i \in I_{j}\right\}}, \quad \forall i \in I .
$$

So, we have $\tilde{f}\left(t, \alpha_{t}^{\varepsilon}, X_{t}^{\varepsilon}\right)=f\left(t, \bar{\alpha}_{t}^{\varepsilon}, X_{t}^{\varepsilon}\right)$, and $M_{t}^{\varepsilon, n}$ is an $\mathcal{F}_{t}^{\varepsilon, n}$-martingale. Thanks to the boundedness and continuity of functions $\varphi_{i}$ and the fixed $\varepsilon^{\prime}$, for $i \leq n, t_{i} \leq s_{1}<s_{2} \leq T$, we have

$$
\begin{aligned}
\mathbb{E} & \left\{\prod_{i=1}^{n} \varphi_{i}\left(\alpha_{t_{i}}^{\varepsilon}, X_{t_{i}}^{\varepsilon}, Y_{t_{i}}^{\varepsilon, n}\right) \int_{0}^{\varepsilon^{\prime}}\left(M_{s_{2}+r}^{\varepsilon, n}-M_{s_{1}+r}^{\varepsilon, n}\right) d r\right\} \\
& =\mathbb{E}\left\{\prod_{i=1}^{n} \varphi_{i}\left(\bar{\alpha}_{t_{i}}^{\varepsilon}, X_{t_{i}}^{\varepsilon}, Y_{t_{i}}^{\varepsilon, n}\right) \int_{0}^{\varepsilon^{\prime}}\left(M_{s_{2}+r}^{\varepsilon, n}-M_{s_{1}+r}^{\varepsilon, n}\right) d r\right\} \\
& =0 .
\end{aligned}
$$

We have already proved that $\left(\bar{\alpha}^{\varepsilon}, X^{\varepsilon}, Y^{\varepsilon, n}, M^{\varepsilon, n}\right)$ converges weakly to $\left(\bar{\alpha}, X, Y^{n}, M^{n}\right)$. Note that $\int_{0}^{\varepsilon^{\prime}}\left(M_{s_{2}+r}^{\varepsilon, n}-M_{s_{1}+r}^{\varepsilon, n}\right) d r$ is a continuous function with respect to $M^{\varepsilon, n}$, we can get $\int_{0}^{\varepsilon^{\prime}}\left(M_{s_{2}+r}^{\varepsilon, n}-M_{s_{1}+r}^{\varepsilon, n}\right) d r$ converges weakly to $\int_{0}^{\varepsilon^{\prime}}\left(M_{s_{2}+r}^{n}-M_{s_{1}+r}^{n}\right) d r$.

This implies that

$$
\mathbb{E}\left\{\prod_{i=1}^{n} \varphi_{i}\left(\bar{\alpha}_{t_{i}}, X_{t_{i}}, Y_{t_{i}}^{n}\right) \int_{0}^{\varepsilon^{\prime}}\left(M_{s_{2}+r}^{n}-M_{s_{1}+r}^{n}\right) d r\right\}=0 .
$$

Dividing by $\varepsilon^{\prime}$, letting $\varepsilon^{\prime} \rightarrow 0$, we get $\mathbb{E}\left\{\prod_{i=1}^{n} \varphi_{i}\left(\bar{\alpha}_{t_{i}}, X_{t_{i}}, Y_{t_{i}}^{n}\right)\left(M_{s_{2}}^{n}-M_{s_{1}}^{n}\right)\right\}=0$, which means that $M_{t}^{n}$ is an $\mathcal{F}_{t}^{\bar{\alpha}, X, Y^{n}}$-martingale. 
Step 4. Identification of the Limit. Suppose that $\left(\bar{Y}^{n}, \bar{Z}^{n}, \bar{W}^{n}\right)$ is the unique solution of the following BSDE:

$$
\begin{aligned}
\bar{Y}_{t}^{n}= & g\left(X_{T}\right)+\int_{t}^{T} f\left(s, \bar{\alpha}_{s}, X_{s}, \bar{Y}_{s}^{n}\right) d s-\int_{t}^{T} \bar{Z}_{s}^{n} d \bar{B}_{s} \\
& -\sum_{j \in \bar{I}} \int_{t}^{T} \bar{W}_{s}^{n}(j) d \tilde{\mathcal{V}}_{s}^{\prime}(j)-\frac{1}{\varepsilon} \int_{t}^{T} D \phi_{\varepsilon}\left(\bar{Y}_{s}^{n}\right) d s
\end{aligned}
$$

where $\bar{B}_{t}$ is a Brownian motion. For its construction, one can see Tao et al. [3].

$$
\begin{aligned}
\mathbb{E}\left|Y_{t}^{n}-\bar{Y}_{t}^{n}\right|^{2}+\mathbb{E}\left[M^{n}-\bar{M}^{n}\right]_{T}-\mathbb{E}\left[M^{n}-\bar{M}^{n}\right]_{t} \\
=2 \mathbb{E} \int_{t}^{T}\left\langle Y_{s}^{n}-\bar{Y}_{s}^{n}, f\left(s, \bar{\alpha}_{s}, X_{s}, Y_{s}^{n}\right)-f\left(s, \bar{\alpha}_{s}, X_{s}, \bar{Y}_{s}^{n}\right)\right\rangle d s \\
\quad-2 \mathbb{E} \int_{t}^{T}\left\langle Y_{s}^{n}-\bar{Y}_{s}^{n}, \frac{1}{\varepsilon} D \phi_{\varepsilon}\left(Y_{s}^{n}\right)-\frac{1}{\varepsilon} D \phi_{\varepsilon}\left(\bar{Y}_{s}^{n}\right)\right\rangle d s,
\end{aligned}
$$

where $\bar{M}_{t}^{n}=\int_{t}^{T} \bar{Z}_{s}^{n} d \bar{B}_{s}+\sum_{j \in \bar{I}} \int_{t}^{T} \bar{W}_{s}^{n}(j) d \tilde{\mathcal{V}}_{s}^{\prime}(j)$, the symbol [·] $]_{t}$ denotes the quadratic variation process. Since $\phi$ is monotone, for $x, z$, it holds that

$$
\left\langle Y_{s}^{n}-\bar{Y}_{s}^{n}, \frac{1}{\varepsilon} D \phi_{\varepsilon}\left(Y_{s}^{n}\right)-\frac{1}{\varepsilon} D \phi_{\varepsilon}\left(\bar{Y}_{s}^{n}\right)\right\rangle \geq 0
$$

Thus, we get $Y_{t}^{n}=\bar{Y}_{t}^{n}$ and $M_{t}^{n}=\bar{M}_{t}^{n}$ a.s. Therefore, the limit process is uniquely determined.

Following the same discussion as that in Proposition 7, we can get Lemma 14 and Lemma 15 easily.

Lemma 14 Under the assumptions above, for fixed $\varepsilon \in(0,1]$, the sequence of processes $\left(Y^{\varepsilon, n}, M^{\varepsilon, n}, U^{\varepsilon, n}\right)_{n}$ converges uniformly in probability to the sequence of processes $\left(Y^{\varepsilon}, M^{\varepsilon}, U^{\varepsilon}\right)$ as $n \rightarrow+\infty$.

Lemma 15 Under the assumptions above, the sequence of processes $\left(Y^{n}, M^{n}, U^{n}\right)_{n}$ converges in probability to the sequence of processes $(Y, M, U)$ as $n \rightarrow+\infty$.

Proof of Theorem 11 Combining the lemmas above, we can prove that $\left(X^{\varepsilon}, Y^{\varepsilon}, M^{\varepsilon}, U^{\varepsilon}\right)$ converges in law to $(X, Y, M, U)$.

\section{Application to the homogenization of multi-valued PDEs}

Then we consider the following multi-valued PDE:

$$
\left\{\begin{array}{l}
\frac{\partial u^{\varepsilon}}{\partial t}(t, i, x)+\mathcal{L} u^{\varepsilon}(t, i, x)+f\left(t, i, x, u^{\varepsilon}(t, i, x)\right) \\
\quad+\sum_{j \neq i, i, \in I} \lambda_{i j}^{\varepsilon}(t)\left(u^{\varepsilon}(t, j, x)-u^{\varepsilon}(t, i, x)\right) \in \partial \phi\left(u^{\varepsilon}(t, i, x)\right), \\
u^{\varepsilon}(T, i, x)=g(x), \quad u^{\varepsilon}(t, i, x) \in \overline{\operatorname{Dom}(\phi)}, \quad x \in \mathbb{R}^{d},
\end{array}\right.
$$


and the following multi-valued PDE:

$$
\left\{\begin{array}{l}
\frac{\partial u}{\partial t}(t, i, x)+\mathcal{L} u(t, i, x)+f(t, i, x, u(t, i, x)) \\
\quad+\sum_{j \neq i, j \in I} \lambda_{i j}(t)(u(t, j, x)-u(t, i, x)) \in \partial \phi(u(t, i, x)), \\
u(T, i, x)=g(x), \quad u(t, i, x) \in \overline{\operatorname{Dom}(\phi)}, \quad x \in \mathbb{R}^{d} .
\end{array}\right.
$$

Define

$$
u^{i}(t, x)=u(t, i, x)
$$

For $\varphi(t, x) \in \mathbb{C}^{1,2}\left([0, T] \times \mathbb{R}^{n}\right)$, we define the following operator:

$$
\mathcal{L}^{i} \varphi(t, x)=\frac{1}{2} \sum_{p, q=1}^{n} a_{p q}(t, i, x) \frac{\partial^{2}}{\partial x_{p} \partial x_{q}} \varphi(t, x)+\sum_{p=1}^{n} b_{p}(t, i, x) \frac{\partial}{\partial x_{p}} \varphi(t, x) .
$$

The viscosity solution of multi-valued PDEs (5.2) is defined as follows, which is similar to Definition 4.1 in [3].

Definition 16 Let $u=\left(u^{1}, \ldots, u^{m}\right)$ belong to $\mathbb{C}\left([0, T] \times \mathbb{R}^{d} ; \mathbb{R}^{m}\right)$. $u$ is said to be a viscosity subsolution (resp. supersolution) of multi-valued PDEs (5.2), if $u^{i}(T, x) \leq g(x)$ for all $i \in$ $I, x \in \mathbb{R}^{d}$ (resp. $\left.u^{i}(T, x) \geq g(x)\right)$ and for all $i \in I,(\bar{t}, \bar{x}) \in(0, T) \times \mathbb{R}^{d}, \varphi \in \mathbb{C}^{1,2}\left((0, T) \times \mathbb{R}^{d} ; \mathbb{R}\right)$ such that $(\bar{t}, \bar{x})$ is a local maximum point (resp. local minimum point) of $u^{i}-\varphi$, it holds that

$$
\begin{aligned}
& -\varphi_{t}(\bar{t}, \bar{x})-\mathcal{L}^{i} \varphi(\bar{t}, \bar{x})-f\left(\bar{t}, i, \bar{x}, u^{i}(t, x)\right)-\sum_{j \neq i, j \in I} \lambda_{i j}(t)\left(u^{j}(\bar{t}, \bar{x})-u^{i}(\bar{t}, \bar{x})\right) \\
& \quad \leq-\phi_{-}^{\prime}\left(u^{i}(\bar{t}, \bar{x})\right) ; \\
& \left(-\varphi_{t}(\bar{t}, \bar{x})-\mathcal{L}^{i} \varphi(\bar{t}, \bar{x})-f\left(\bar{t}, i, \bar{x}, u^{i}(\bar{t}, \bar{x})\right)-\sum_{j \neq i, j \in I} \lambda_{i j}(t)\left(u^{j}(\bar{t}, \bar{x})-u^{i}(\bar{t}, \bar{x})\right)\right. \\
& \left.\quad \geq-\phi_{+}^{\prime}\left(u^{i}(\bar{t}, \bar{x})\right)\right) \quad(\text { resp. }) ;
\end{aligned}
$$

$u$ is a viscosity solution of multi-valued PDE (5.2) if it is both a viscosity subsolution and a viscosity supersolution of PDE (5.2).

Consider the following FBSDE:

$$
\left\{\begin{array}{l}
d X_{s}^{t, i, x}=b\left(s, \alpha_{s}^{t, i}, X_{s}^{t, i, x}\right) d s+\sigma\left(s, \alpha_{s}^{t, i}, X_{s}^{t, i, x}\right) d B_{s}, \\
X_{t}^{t, i, x}=x, \quad \alpha_{t}^{t, i}=i, \quad t \leq s,
\end{array}\right.
$$

and

$$
\left\{\begin{aligned}
-d Y_{s}^{t, i, x}= & f\left(s, \alpha_{s}^{t, i}, X_{s}^{t, i, x}, Y_{s}^{t, i, x}\right) d s-Z_{s}^{t, i, x} d B_{s} \\
& -\sum_{j \in I} W_{s}^{t, i, x}(j) d \tilde{\mathcal{V}}_{s}(j)+\partial \phi\left(Y_{s}^{t, i, x}\right) d s, \\
Y_{T}^{t, i, x}= & g\left(X_{T}^{t, i, x}\right), \quad t \leq s \leq T .
\end{aligned}\right.
$$


Define

$$
u^{i}(t, x) \triangleq Y_{t}^{t, i, x}
$$

Note that $u=u\left(u^{1}, \ldots, u^{m}\right)$ defined above is a deterministic measurable function.

For $f+\nabla \phi$ satisfying the conditions of Lemma 4.2 of [3], we have the following result.

Lemma 17 For all $(t, i, x) \in[0, T] \times I \times \mathbb{R}^{d}$, we have a.s.

$$
\begin{aligned}
& Y_{s}^{t, i, x}=u\left(s, \alpha_{s}^{t, i}, X_{s}^{t, i, x}\right), \\
& W_{s}^{t, i, x}(j)=u\left(s, j, X_{s}^{t, i, x}\right)-u\left(s, \alpha_{s_{-}}^{t, i}, X_{s}^{t, i, x}\right),
\end{aligned}
$$

when $j \neq \alpha_{s-}^{t, i}$.

Now, we propose the viscosity solution for (5.6).

Theorem 18 Under the assumptions above, the function $u^{i}(t, x)$ defined by (5.6) is the viscosity solution of Eq. (5.2).

Proof For $\delta \in] 0,1]$, let $\left(Y_{s}^{t, i, x, \delta}, Z_{s}^{t, i, x, \delta}, W_{s}^{t, i, x, \delta}\right)_{t \leq s \leq T}$ be the solution of the following multivalued BSDE:

$$
\begin{aligned}
Y_{s}^{t, i, x, \delta}= & g\left(X_{T}^{t, i}\right)+\int_{t}^{T} f\left(s, \alpha_{s}^{t, i}, X_{s}^{t, i, x, \delta}, Y_{s}^{t, i, x, \delta}\right) d s-\int_{t}^{T} Z_{s}^{t, i, x, \delta} d B_{s} \\
& -\sum_{j \in I} \int_{t}^{T} W_{s}^{t, i, x, \delta}(j) d \tilde{\mathcal{V}}_{s}(j)-\int_{t}^{T} \frac{1}{\delta} D \phi_{\delta}\left(Y_{s}^{t, i, x, \delta}\right) d s .
\end{aligned}
$$

From Theorem 4.3 of [3], we obtain

$$
u_{\delta}^{i}(t, x) \triangleq Y_{t}^{t, i, x, \delta}, \quad t \in[0, T], x \in \mathbb{R}^{d}
$$

is a viscosity solution of the following PDE:

$$
\left\{\begin{array}{l}
\frac{\partial u_{\delta}^{i}}{\partial t}(t, x)+\mathcal{L} u_{\delta}^{i}(t, x)+f\left(t, i, x, u_{\delta}^{i}(t, x)\right)+\sum_{j \neq i, j \in I} \lambda_{i j}^{\delta}(t)\left(u_{\delta}^{j}(t, x)-u_{\delta}^{i}(t, x)\right) \\
\quad=\frac{1}{\delta} D \phi_{\delta}\left(u_{\delta}^{i}(t, x)\right), \\
u_{\delta}^{i}(T, x)=g(x), \quad u_{\delta}^{i}(t, x) \in \overline{\operatorname{Dom}(\phi)}, \quad x \in \mathbb{R}^{d} .
\end{array}\right.
$$

By the conclusion in Sect. 4, we have

$$
\left|u_{\delta}^{i}(t, x)-u^{i}(t, x)\right| \leq \mathbb{E}\left(\sup _{s \in[t, T]}\left|Y_{s}^{t, i, x, \delta}-Y_{s}^{t, i, x}\right|\right) \rightarrow 0, \quad \text { as } \delta \rightarrow 0,
$$

for all $(t, i, x) \in[0, T] \times I \times \mathbb{R}^{d}$. Next, we prove that $u$ is a viscosity subsolution of PDE (5.2).

There exist the sequences

$$
\left\{\begin{array}{l}
\delta_{n} \searrow 0 \\
\left(t_{n}, x_{n}\right) \in[0, T] \times \mathbb{R}^{d}
\end{array}\right.
$$


such that

$$
\left(t_{n}, x_{n}, u_{\delta_{n}}^{i}\left(t_{n}, x_{n}\right)\right) \rightarrow\left(t, x, u^{i}(t, x)\right), \quad \text { as } n \rightarrow+\infty
$$

For any $n$, we have

$$
\begin{aligned}
& -\frac{\partial u_{\delta_{n}}^{i}}{\partial t_{n}}\left(t_{n}, x_{n}\right)-\mathcal{L} u_{\delta_{n}}^{i}\left(t_{n}, x_{n}\right)-f\left(t_{n}, i, x_{n}, u_{\delta_{n}}^{i}\left(t_{n}, x_{n}\right)\right) \\
& \quad-\sum_{j \neq i, j \in I} \lambda_{i j}^{\delta_{n}}(t)\left(u_{\delta_{n}}^{j}\left(t_{n}, x_{n}\right)-u_{\delta_{n}}^{i}\left(t_{n}, x_{n}\right)\right) \\
& \quad \leq \frac{1}{\delta_{n}} D \phi_{\delta_{n}}\left(u_{\delta_{n}}^{i}\left(t_{n}, x_{n}\right)\right) .
\end{aligned}
$$

Let $y \in \operatorname{Dom}(\phi), y<u^{i}(t, x)$, by (5.8), the uniform convergence $u_{\delta} \rightarrow u$ on compacts indicates that there exists $n_{0}>0$ such that $y<u_{\delta_{n}}^{i}\left(t_{n}, x_{n}\right)$ for $n \geq n_{0}$. Multiplying Eq. (5.10) by $u_{\delta_{n}}^{i}\left(t_{n}, x_{n}\right)-y$, we obtain

$$
\begin{aligned}
& \left\{-\frac{\partial u_{\delta_{n}}^{i}}{\partial t_{n}}\left(t_{n}, x_{n}\right)-\mathcal{L} u_{\delta_{n}}^{i}\left(t_{n}, x_{n}\right)-f\left(t_{n}, i, x_{n}, u_{\delta_{n}}^{i}\left(t_{n}, x_{n}\right)\right)\right. \\
& \left.\quad-\sum_{j \neq i, j \in I} \lambda_{i j}(t)\left(u_{\delta_{n}}^{j}\left(t_{n}, x_{n}\right)-u_{\delta_{n}}^{i}\left(t_{n}, x_{n}\right)\right)\right\}\left(u_{\delta_{n}}^{i}\left(t_{n}, x_{n}\right)-y\right) \\
& \leq \phi(y)-\phi\left(J_{\delta_{n}}\left(u_{\delta_{n}}^{i}\left(t_{n}, x_{n}\right)\right)\right),
\end{aligned}
$$

passing to $\liminf _{n \rightarrow+\infty}$ on both sides of Eq. (5.11), we have that, for all $y<u^{i}(t, x)$,

$$
\begin{aligned}
& \left\{-\frac{\partial u^{i}}{\partial t}(t, x)-\mathcal{L} u^{i}(t, x)-f\left(t, i, x, u^{i}(t, x)\right)\right. \\
& \left.-\sum_{j \neq i, j \in I} \lambda_{i j}(t)\left(u^{j}(t, x)-u^{i}(t, x)\right)\right\}\left(u^{i}(t, x)-y\right) \\
& \leq \phi(y)-\phi\left(u^{i}(t, x)\right) .
\end{aligned}
$$

It follows that

$$
\begin{aligned}
& -\frac{\partial u^{i}}{\partial t}(t, x)-\mathcal{L} u^{i}(t, x)-f\left(t, i, x, u^{i}(t, x)\right)-\sum_{j \neq i, j \in I} \lambda_{i j}(t)\left(u^{j}(t, x)-u^{i}(t, x)\right) \\
& \quad \leq-\phi_{-}^{\prime}\left(u^{i}(t, x)\right),
\end{aligned}
$$

i.e., $\mathrm{u}$ is a viscosity subsolution of Eq. (5.2). By similar arguments, we can show that $u$ is a viscosity supersolution of Eq. (5.2). As the same idea of Theorem 4.2 of Pardoux and Răşcanu [6], the uniqueness comes out.

Corollary 19 Let $u^{\varepsilon}(t, x)$ be the unique viscosity solution for the multi-valued PDE (5.1). Then, for $t \in[0, T], x \in \mathbb{R}^{d}, u^{\varepsilon}$ converges to the unique viscosity solution $u(t, x)$ of (5.2) as $\varepsilon \rightarrow 0$. 


\section{Acknowledgements}

The authors express their sincerest thanks to the reviewers for their valuable comments, which further improved the conclusion and proof process of the article.

Funding

The work is supported by NSFC (NO. 11871076) and the Natural Science Foundation of Anhui Province (NO. KJ2019A0976).

\section{Availability of data and materials}

Not applicable.

\section{Competing interests}

The authors declare that they have no competing interests.

\section{Authors' contributions}

The two authors completed the discussion of the original structure and results together. RD completed the drafting of the manuscript. All authors read and approved the final manuscript.

\section{Author details}

'Department of Basic, Wuhu Institute of Technology, Wuhu, China. ${ }^{2}$ School of Mathematics and Statistics, Anhui Normal University, Wuhu, China.

\section{Publisher's Note}

Springer Nature remains neutral with regard to jurisdictional claims in published maps and institutional affiliations.

Received: 21 January 2019 Accepted: 15 November 2019 Published online: 16 December 2019

\section{References}

1. Siu, T.K.: Integration by parts and martingale representation for a Markov chain. Abstr. Appl. Anal. 2014(1), 189 (2014)

2. Lu, W., Ren, Y.: Mean-field backward stochastic differential equations on Markov chains. Bull. Korean Math. Soc. 54(1), $17-28(2017)$

3. Tao, R., Wu, Z., Zhang, Q.: BSDEs with regime switching: weak convergence and applications. J. Math. Anal. Appl. 407(1), 97-111 (2013)

4. Wu, Z., Zhang, F.: BDSDEs with locally monotone coefficients and Sobolev solutions for SPDEs. J. Differ. Equ. 251(4-5), 759-784 (2011)

5. Wei, L., Wu, Z., Zhao, H.: Sobolev weak solutions of the Hamilton-Jacobi-Bellman equations. SIAM J. Control Optim. 52(3), 1499-1526 (2014)

6. Pardoux, E., Răscanu, A.: Backward stochastic differential equations with subdifferential operator and related variational inequalities. Stoch. Process. Appl. 76(2), 191-215 (1998)

7. Yang, F., Ren, Y., Hu, L.: Multi-valued backward stochastic differential equations driven by G-Brownian motion and its applications. Math. Methods Appl. Sci. 40(13), 4696-4708 (2017)

8. Guo, $Z: L^{p}(p \geq 2)$-strong convergence in averaging principle for multivalued stochastic differential equation with non-Lipschitz coefficients. Adv. Differ. Equ. 2018, 386 (2017)

9. Malinowski, M.T., Agarwal, R.P.: On solutions set of a multivalued stochastic differential equation. Czechoslov. Math. J. 67(1), 11-28 (2017)

10. Brézis, H.: Opérateurs Maximaux Monotones et Semi-Groupes de Contractions dans les Espaces de Hilbert. North Holland, Amsterdam (1973)

11. Crépey, S., Matoussi, A.: Reflected and doubly reflected BSDEs with jumps: a priori estimates and comparison. Ann. Appl. Probab. 18(5), 2041-2069 (2008)

12. $\mathrm{Wu}, \mathrm{Z}$., $\mathrm{Yu}, \mathrm{Z}$ :. Dynamic programming principle for one kind of stochastic recursive optimal control problem and Hamilton-Jacobi-Bellman equations. SIAM J. Control Optim. 47(5), 2616-2641 (2007)

13. Yin, G., Zhang, Q:. Continuous-Time Markov Chains and Applications: A Two-Time-Scale Approach. Springer, New York (2012)

14. Meyer, P.A., Zheng, W.A.: Tightness criteria for laws of semimartingales. Ann. Inst. Henri Poincaré Probab. Stat. 20(4), 353-372 (1984)

15. Billingsley, P.: Convergence of Probability Measures. Wiley, New York (1968) 